\title{
Metodología de toma de decisiones para la selección de subcontratos en la industria de la construcción
}

\section{Methodology of decision-making for the selection of subcontracts in the construction industry}

Fecha de entrega: 19 de marzo 2018 Fecha de aceptación: 17 de octubre 2018

\section{Andrés Crisóstomo y Rodrigo F. Herrera}

Escuela de Ingeniería Civil, Facultad de Ingeniería, Pontificia Universidad Católica de Valparaíso, Avenida Brasil 2147, Valparaíso, Chile, andrescrisostomoriquelme@gmail.com, rodrigo.herrera@pucv.cl

Una selección de subcontratistas errónea en la industria de la construcción causa problemas críticos como: retrasos en los proyectos, calidad deficiente de los trabajos y costos adicionales. Este trabajo entrega una herramienta para la selección de subcontratos, ésta se traduce en una metodología de toma de decisiones para seleccionar el subcontrato más adecuado para cada proyecto. Para lograr el objetivo, primero se determinan las prácticas actuales de subcontratación a través de una encuesta que fue contestada por cincuenta empresas del rubro. Posteriormente, se analizan dos métodos de toma de decisiones (WRC y CBA) en una simulación de toma de decisiones que fue realizada a diez empresas del rubro de forma presencial. Los resultados fueron concluyentes, en doce de las quince simulaciones el método WRC destacó al contrastarse con el método CBA, por lo cual se define al método WRC como el más adecuado para este tipo de selección. Finalmente, se presenta la metodología de toma de decisiones como una herramienta de optimización del proceso de selección de subcontratistas, en ésta se incluyen aspectos como: recomendaciones antes del proceso de selección y la estructura en detalle para desarrollar correctamente el método WRC.

Palabras clave: subcontratación, industria de la construcción, toma de decisiones, WRC, CBA
A poor selection of subcontractors in the construction industry causes critical problems such as: delays in projects, poor quality of work and additional costs. This paper provides a tool for selection of subcontracts, which translates into a decision-making methodology to select the most suitable subcontract for each project. To achieve this objective, first the current subcontracting practices are determined through a survey that was answered by fifty companies of the sector. Subsequently, the decisionmaking methods (WRC and CBA) are analyzed in a decision-making simulation that was carried out to ten companies in the field. The results were conclusive, in twelve of the fifteen simulations, WRC method highlighted when contrasting with the CBA method, so the WRC method is defined as the most appropriate for this type of selection. Finally, the decision-making methodology is presented as an optimization tool for the selection process of subcontractors, which includes aspects such as: recommendations before the selection process and structure in detail to correctly develop the WRC method.

Keywords: subcontracts, construction industry, decision-making, $W R C, C B A$

\section{Introducción}

La subcontratación laboral, entre sus variadas definiciones, puede enunciarse como el proceso económico en el cual una empresa determinada mueve o destina recursos orientados a cumplir ciertas tareas a una empresa externa por medio de un contrato (Uquillas, 2007). Este concepto comienza a ganar credibilidad al inicio de los años 70 , dando inicio a una nueva estrategia de negocios en los mercados globales. La OIT (2007) plantea que dicha estrategia de negocios ha tenido un sostenido crecimiento global en las últimas décadas, debido principalmente al proceso de globalización de la economía y la fuerte competencia internacional. En la actualidad, la industria chilena de la construcción está cada vez más enfocada a que empresas de mayor tamaño subcontraten servicios de empresas constructoras menores y más especializadas en determinados nichos constructivos (Alvarado y Spolmann, 2009). Por lo cual, se hace imprescindible contar con herramientas de toma de decisiones para seleccionar los 
subcontratos más beneficiosos para las empresas en cada proyecto. La decisión de subcontratar conlleva variados aspectos positivos como negativos, una mala selección de éstos, por ejemplo, puede causar problemas críticos en términos de tiempo, traduciéndose en retrasos del proyecto (Sambasivan y Soon, 2007; Odeh y Battaineh, 2002). Según Uquillas (2007), la selección de proveedores y/o subcontratistas es uno de los tres aspectos fundamentales para lograr un buen proyecto.

En la presente investigación, se busca desarrollar una metodología de toma de decisiones para seleccionar subcontratistas en la industria de la construcción, entregando una nueva perspectiva y herramienta para la optimización de dicho proceso de selección. Para lograr dicho objetivo, se necesita inicialmente identificar las prácticas actuales de selección de subcontratistas en el rubro para determinar los parámetros más relevantes que usan las empresas a la hora de escoger el subcontrato más beneficioso. Posteriormente, considerando dichos parámetros se diseñó un experimento de simulación de toma de decisiones, en el cual un grupo de trabajo que interviene directamente en el proceso de subcontratación será sometido a casos hipotéticos de subcontratación en distintos escenarios, aplicando distintos métodos de toma de decisiones. Una vez terminado el diseño del experimento de simulación, se procedió a implementar dicha simulación con profesionales del sector de la construcción. En dicha instancia se midieron los indicadores más influyentes al momento de tomar las decisiones para la elección de un subcontratista determinado. Finalmente, se propone una metodología de toma de decisiones en la industria de la construcción.

En la primera parte de este trabajo se plantea el problema de investigación, donde se hace énfasis al hecho que una mala selección de subcontratistas en el rubro causa problemas en los proyectos. Se enuncian los métodos de toma de decisiones, se presentan y analizan para luego proponer la utilización de éstos para la optimización de la selección de dichos subcontratistas. Después se presenta el diagnóstico de las prácticas actuales de subcontratación, el cual se traduce en una encuesta contestada por 50 empresas del rubro. Posteriormente, se presenta el diseño del experimento de simulación, en el cual se pretende contrastar el método habitual de selección de subcontratistas con dos métodos multicriterios de toma de decisiones. Finalmente, se presenta un análisis estadístico de los indicadores recogidos en dicho experimento y se concluye dando un manual para la optimización de la selección de los subcontratistas en la industria de la construcción.

\section{Antecedentes}

\section{Toma de decisiones en la industria de la construcción}

Generalmente, cuando los proyectos se retrasan, éstos se extienden en tiempo o aceleran su ritmo de trabajo, incurriendo en ambos casos, a costos adicionales para la empresa (Sambasivan y Soon, 2007). Según Assaf y AlHejji (2006), en la industria de la construcción, el retraso puede definirse como el tiempo excedido de ejecución más allá de la fecha de finalización en un contrato, o más allá de la fecha en que las partes acordaron entregar un proyecto. En algunos casos, para el contratista, el retraso significa también sobrecostos por un tiempo mayor de trabajo, además de mayores costos en materiales y mano de obra.

Una vasta cantidad de autores han detectado las causas de los retrasos en las obras de construcción en distintos países a lo largo del mundo. Según Mezher y Tawil (1998), los dueños de las empresas de construcción del Líbano, indicaron que el mayor factor de retrasos fue una mala programación por parte de los subcontratistas. Ogunlana et al. (1996) estudiaron las causas de los retrasos en los proyectos de construcción en Tailandia, concluyeron que uno de los tres grandes ámbitos de problemas eran los problemas causados por la incompetencia de los contratistas. Assaf et al. (1995) estudiaron las causas de los retrasos en los grandes proyectos de construcción en Arabia Saudita, concluyeron que una de las mayores causas de retrasos eran los conflictos producidos por la programación de trabajo por parte de los subcontratos. De acuerdo a los resultados de Sambasivan y Soon (2007), los problemas con los subcontratistas fue una de las primeras diez causas de retrasos en la construcción en Malasia. Típicamente, en los grandes proyectos existen variados subcontratistas trabajando para un mismo contratista, si estos contratistas no tienen una adecuada experiencia o capacidad los proyectos pueden verse retrasados. Según el estudio de Odeh y Battaineh (2002), uno de los diez factores que causan mayores retrasos en la construcción en Jordania, son los retrasos causados por sus propios subcontratistas. Estos autores señalan que los contratistas y las consultoras que respondieron dicho estudio indican 
que los niveles de retrasos alcanzados por causa de los retrasos de los subcontratistas son preocupantes, los cuales se pueden atribuir a la inadecuada experiencia de los subcontratistas, además de una mala planificación y coordinación. Es por lo anterior, que resulta fundamental que las empresas constructoras tengan una metodología de toma de decisiones para seleccionar a sus subcontratos.

\section{Métodos de toma de decisiones}

La toma de decisiones puede definirse como el estudio para identificar y elegir alternativas basadas en valores y preferencias del decisor (Harris, 2012). El mismo autor, termina enunciando que es un proceso en el cual se reduce la incertidumbre sobre ciertas alternativas para permitir una elección razonable entre ellas. El proceso de toma de decisiones envuelve una serie de pasos: identificar los problemas, determinar las preferencias, evaluar las alternativas y determinar la mejor alternativa (Kleindorfer et al., 1993). Según Tzeng y Huang (2011), cuando la toma de decisiones posee sólo un criterio para su selección, es un proceso extremadamente intuitivo, ya que sólo se debe elegir la alternativa con el mayor puntaje de preferencia. Sin embargo, cuando la toma de decisiones posee múltiples criterios surgen muchos problemas: cómo definir los pesos de cada criterio, qué dependencia tiene una preferencia sobre otra, qué conflictos existen entre los criterios, entre otros. Dichos problemas parecen complicar la decisión y se hace necesario usar métodos más sofisticados como los métodos multicriterios de toma de decisiones. A continuación, se presentan algunos métodos de toma de decisiones multicriterios para seleccionar de mejor manera los subcontratos en el rubro de la construcción, esperando que con su utilización exista una disminución de los retrasos en los proyectos y aumente la eficiencia del rubro.

\section{Weighting Rating and Calculating WRC}

Según Arroyo et al. (2016), el método WRC es extensamente usado en las industrias de la arquitectura, ingeniería y construcción AIC. Por ejemplo, Sabapathy y Maithel (2013) usaron WRC para crear un ranking para escoger materiales para paredes en India, y Tatum (1984) describe el uso de WRC en un amplio rango de decisiones. La Figura 1 muestra la metodología, donde el primer paso es identificar las alternativas de selección, las cuales garantizan alcanzar el objetivo propuesto. Segundo, se deben identificar los factores y criterios de evaluación, los cuales para el decisor son claves en su decisión. Tercero, se deben dar pesos $w_{\mathrm{i}}$ a los factores directamente, éstos indican la importancia de cada factor para el decisor. Como restricción, la suma de los pesos debe ser $100 \%$. Cuarto, se debe calificar las alternativas por cada factor $u_{\mathrm{i}}$, dicha calificación dependerá de las características de cada alternativa. En el último paso, se calcula el valor de cada alternativa de la siguiente manera: $\sum w_{\mathrm{i}} u_{\mathrm{i}}$, siendo $w_{\mathrm{i}}$ el peso de los factores y $u_{\mathrm{i}}$ las calificaciones de los factores en cada alternativa. Finalmente, el mayor valor de todas las alternativas será la mejor opción según todas las decisiones anteriores tomadas en el desarrollo del método.

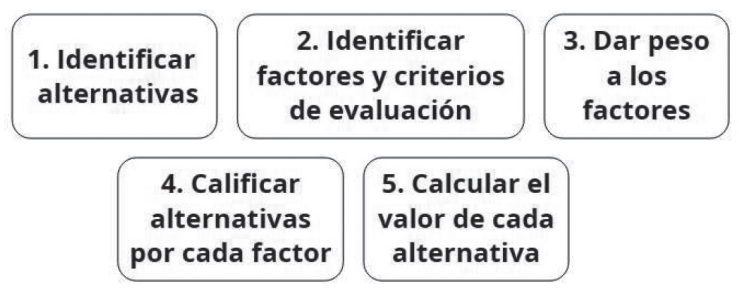

Figura 1: Pasos del método WRC (Arroyo et al., 2014).

\section{Choosing By Advantage CBA}

Según Arroyo et al. (2016), el método CBA es mayormente usado en comunidades Lean. Por ejemplo, Grant y Jones (2008) usan CBA para seleccionar los diseños de techos verdes, y Nguyen et al. (2009) usan CBA para seleccionar sistemas de muros. En esta metodología, mostrada en la Figura 2, el primer paso es identificar las alternativas de selección, las cuales deben tener ventajas claras entre ellas. Segundo, se identifican los factores a evaluar, los cuales son claves para el decisor. Tercero, se debe definir el criterio deseado para cada factor, éstos pueden ser criterios deseados u obligatorios. Cuarto, se deben resumir los atributos de cada alternativa por cada factor, dicho resumen dependerá de las características de cada alternativa. Quinto, se debe decidir las ventajas de cada alternativa sobre la ventaja más desfavorable. Sexto, se debe decidir la importancia de cada ventaja, en una escala de 0 a 100, siendo 100 la mayor importancia. Finalmente, se debe evaluar el costo de cada alternativa y su respectiva importancia para tomar la decisión. Finalmente, se debe construir un gráfico con ejes de costo y de importancia total, con dicho apoyo se debe tomar la decisión final. 


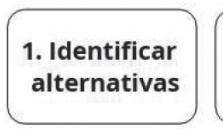

\section{Definir los factores de evaluación}

5. Decidir ventaja de cada alternativa
3. Definir criterio deseado para cada factor
4. Resumir atributos de alternativas

Figura 2: Pasos del método CBA (Schöttle et al., 2015)

\section{Metodología de investigación}

La Figura 3 representa la metodología de investigación seguida para la selección de subcontratistas en la industria de la construcción.

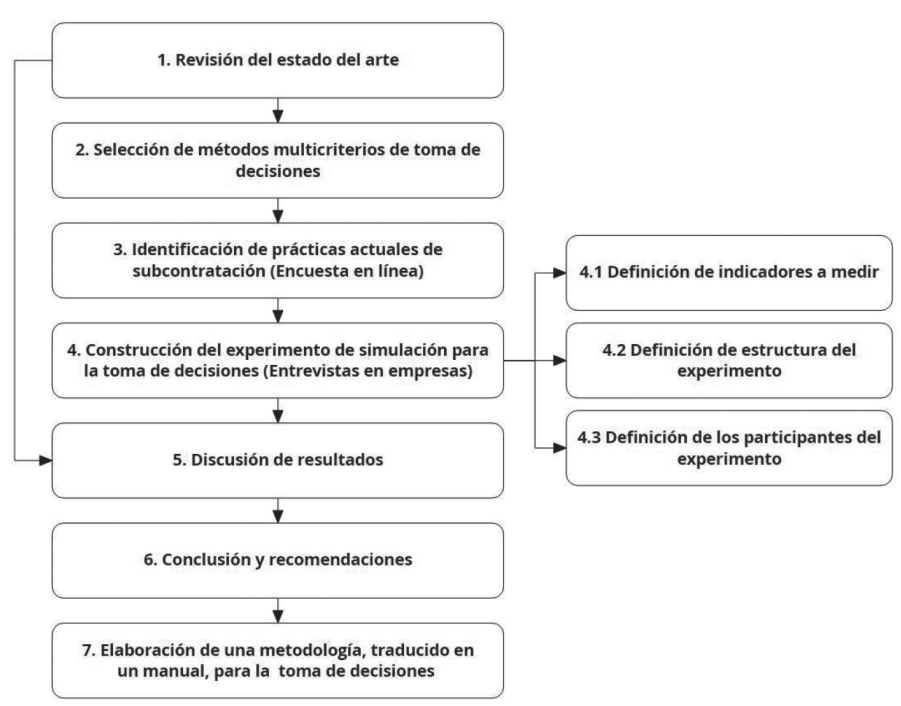

Figura 3: Metodología de investigación

En primer lugar, se hizo una extensa revisión del estado del arte de la subcontratación en la industria de la construcción. Luego, elegir los métodos multicriterio de toma de decisiones como una posible optimización al proceso de selección de subcontratistas en el rubro. Posteriormente, se diseña un set de preguntas dirigidas a profesionales del rubro de la construcción para identificar las prácticas actuales de subcontratación. Seguidamente, se diseña el experimento de simulación para la toma de decisiones, en éste se pretende contrastar el método habitual de toma de decisiones de las empresas (S/M) con los dos métodos de toma de decisiones propuestos como optimización. Para dicho diseño, inicialmente se definen los indicadores que serán medidos, luego se define la estructura que seguirá y finalmente se definirán los participantes de la simulación.

A continuación, se realizó un análisis estadístico de todos los indicadores medidos en dicho experimento de

simulación. En éste, se pretende dirimir qué método se desempeñó mejor en la experiencia para luego proponerlo como herramienta para la selección de subcontratistas en la industria.

Finalmente, se elaboró la metodología para la selección de subcontratistas en la industria de la construcción, ésta se verá traducida en un manual para las empresas. Dicho manual, incluye recomendaciones antes del proceso de selección de los subcontratistas y los respectivos pasos del método de toma de decisiones que tuvo mejor desempeño en la investigación.

\section{Diagnóstico de las prácticas actuales de subcontratación}

Como punto de partida de investigación y para conocer las prácticas actuales de subcontratación en el rubro, se llevó a cabo una encuesta dirigida a profesionales con un cargo relevante a la hora de tomar decisiones para la selección de subcontratistas en sus respectivas empresas. Dicha encuesta fue contestada por 50 profesionales, con cargos como: profesionales de obra, administradores de obra, asesores de ingeniería, jefes de ingeniería, ingenieros de proyectos, directores, gerentes y gerentes generales. Para conocer los parámetros más relevantes para la selección de subcontratistas que tienen las empresas en la industria de la construcción, se preguntó por la característica que más valoran al momento de subcontratar. En la Figura 4, se pueden observar las respuestas a dicha interrogante.

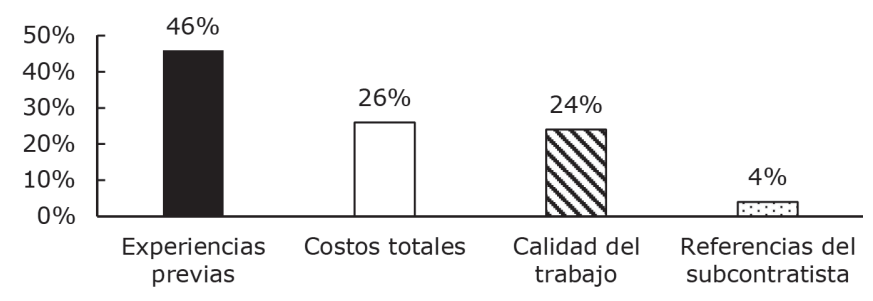

Figura 4: Porcentaje de empresas encuestadas según la característica más valorada al momento de subcontratar

Con dichas cifras, podemos observar que el $46 \%$ de las empresas encuestadas consideran como característica preponderante la experiencia previa con el subcontratista, es decir, si conocen y han trabajado en ocasiones anteriores con ellos. Luego, con $26 \%$ y $24 \%$ lo siguen la calidad de trabajos anteriores y los costos totales que cobran dichos subcontratistas, respectivamente. Podemos afirmar 
entonces, que la mitad de las empresas encuestadas se fijan en los costos y la calidad de trabajos de los subcontratistas a la hora de seleccionarlos. Finalmente, con un $4 \%$ se valora las referencias del subcontratista por otras personas o colegas, dicho valor refleja que se valora más la experiencia propia con los subcontratistas que la referencias de otros. Luego, se consultó por el modo en que seleccionan el subcontratista, si decide una persona en particular, si deciden junto a un grupo de trabajo, si lo decide una oficina central u otra opción. Dichas respuestas se observan en la Figura 5.

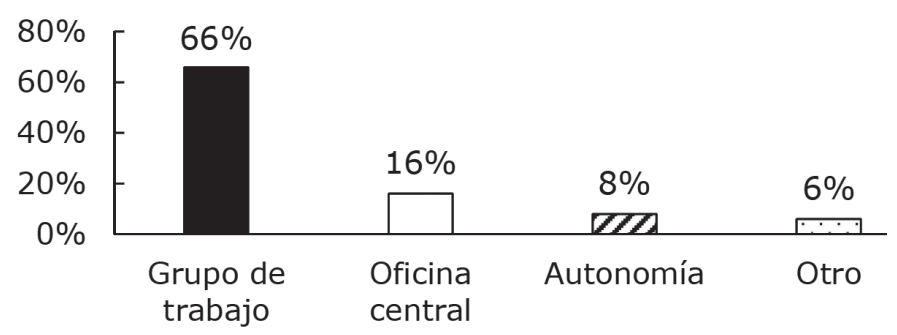

Figura 5: Porcentaje de empresas encuestadas según el modo de seleccionar a los subcontratistas

Se afirma entonces, que la opinión del grupo de trabajo es ampliamente valorada, ya que más de la mitad de las empresas analizadas (66\%) toma las decisiones de los subcontratos en conjunto y no son exclusivas de una persona u oficina central. Finalmente, para profundizar en el tema del mal desempeño de los subcontratistas en el rubro, se preguntó cuáles eran los problemas más comunes de dichos subcontratistas. Los resultados se pueden observar en la Figura 6.

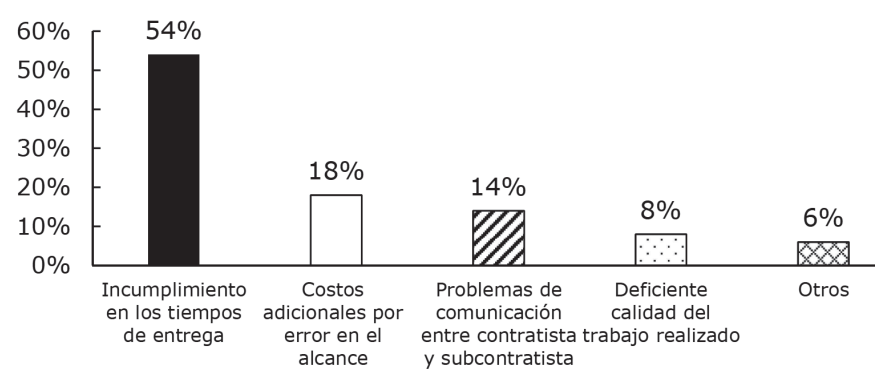

Figura 6: Porcentaje de empresas encuestadas según los problemas más comunes de los subcontratistas

Se puede observar que el parámetro más recurrente (54\%) en este ítem, fue el incumplimiento en los tiempos de entrega, lo cual indica que en el rubro de la construcción el parámetro del tiempo es el más crítico.

\section{Simulación de toma de decisiones}

Se realizó un experimento de simulación de toma de decisiones con el fin de determinar cuál es el método multicriterio que se desempeña mejor al seleccionar subcontratistas en la industria de la construcción. La Figura 7 representa la estructura del experimento de simulación de toma de decisiones que se implementó en la presente investigación.

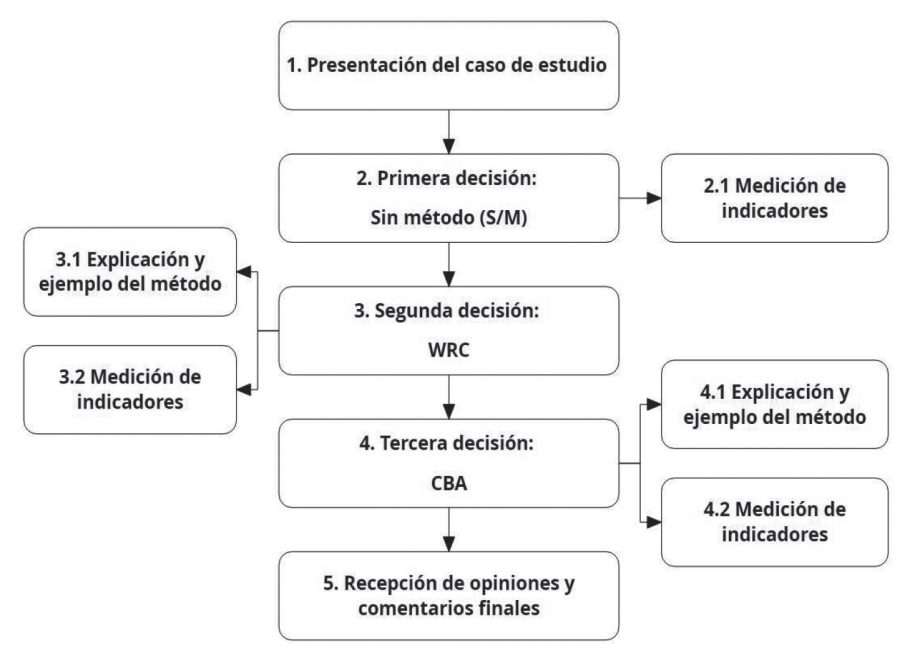

Figura 7: Estructura del experimento de simulación

En primer lugar, se procede a presentar y explicitar el caso de estudio que será aplicado en las tres decisiones posteriores. Dicho caso de estudio, se trata de la construcción de un edificio de veinte pisos en la ciudad de Viña del Mar. Seguidamente, los participantes deben tomar la primera decisión sin ningún método, en este caso, individualmente deben seleccionar el mejor subcontrato de la partida de acero entre las tres ofertas de subcontratos que se entregan como información adicional. Una vez todos los participantes hayan tomado su decisión, se procede a indicarles que deben llegar a un consenso grupal acerca de dicha selección, una vez concluya esta etapa de discusión, se procede a medir los indicadores mostrados en la Tabla 1. Luego, a los participantes se les presenta el primer método multicriterio para la toma de decisiones llamado WRC. En este punto, se les explica cómo funciona el método, cuáles son sus pasos más relevantes y se desarrolla un ejemplo en conjunto. Luego, los participantes deben tomar la segunda decisión, en este caso, individualmente deben seleccionar el mejor subcontrato de la partida de moldajes entre las tres ofertas de subcontratos que se entregan como información 
adicional. Una vez todos los participantes hayan tomado su decisión, se procede a indicarles que deben llegar a un consenso grupal acerca de dicha selección, una vez concluya esta etapa de discusión, se procede a medir los indicadores (Tabla 1).

Posteriormente, a los participantes se les presenta el segundo método multicriterio para la toma de decisiones llamado CBA. En este punto, se les explica cómo funciona el método, cuáles son sus pasos más relevantes y se desarrolla un ejemplo en conjunto. Luego, los participantes deben tomar la tercera decisión, en este caso, individualmente deben seleccionar el mejor subcontrato de la partida de hormigón entre las tres ofertas de subcontratos que se entregan como información. Una vez todos los participantes hayan tomado su decisión, se procede a indicarles que deben llegar a un consenso grupal acerca de dicha selección, una vez concluya esta etapa de discusión, se procede a medir los indicadores (Tabla 1). Finalmente, los participantes entregan sus opiniones y comentarios finales acerca de la experiencia que acaban de realizar y los métodos propuestos.

Los participantes del experimento de simulación para la toma de decisiones fueron diez empresas del rubro de la construcción, específicamente, diez grupos de trabajo de dos a tres personas por sesión. Entre dichas empresas, se encuentran: seis constructoras y cuatro oficinas de ingeniería en la Región Metropolitana y de Valparaíso. De los 10 grupos de trabajo, se contó con la participación de profesionales del rubro como: administradores de obra, jefes de ingeniería, dueños de constructoras, ingenieros de proyectos, ingenieros de oficinas técnicas, gerentes y gerentes generales. Las constructoras y oficinas de ingeniería se dedicaban a proyectos de edificación de envergadura similar.

\section{Discusión de resultados}

El primer indicador medido fue la satisfacción personal, dicho indicador mide qué tan satisfechos se sienten con la decisión grupal respecto a su decisión individual. Su escala de medición es de uno a diez, siendo uno el menor grado de satisfacción y diez el mayor grado de satisfacción. Los resultados se presentan en la Tabla 2 y representan la mediana de las satisfacciones de los trabajadores en cada empresa.

Tabla 1: Indicadores medidos durante la simulación

\begin{tabular}{|c|c|c|c|}
\hline Satisfacción personal & $\begin{array}{l}\text { Nivel de satisfacción con la } \\
\text { decisión grupal respecto a su } \\
\text { decisión individual }\end{array}$ & $\begin{array}{l}\text { De uno a diez. } \\
\text { Siendo uno el más bajo y diez } \\
\text { el más alto. }\end{array}$ & $\begin{array}{l}\text { Posterior a la decisión tomada } \\
\text { con } \mathrm{S} / \mathrm{M}, \mathrm{WRC} \text { y CBA. }\end{array}$ \\
\hline Frustración personal & $\begin{array}{l}\text { Nivel de frustración con la } \\
\text { decisión grupal respecto a su } \\
\text { decisión individual }\end{array}$ & $\begin{array}{l}\text { De uno a diez. } \\
\text { Siendo uno el más bajo y diez } \\
\text { el más alto. }\end{array}$ & $\begin{array}{l}\text { Posterior a la decisión tomada } \\
\text { con } \mathrm{S} / \mathrm{M}, \mathrm{WRC} \text { y CBA. }\end{array}$ \\
\hline $\begin{array}{l}\text { Probabilidad de } \\
\text { implementación del método }\end{array}$ & $\begin{array}{l}\text { Probabilidad de implementar } \\
\text { el método en su empresa }\end{array}$ & $\begin{array}{l}\text { De cero a uno. } \\
\text { Siendo cero el más bajo y uno } \\
\text { el más alto. }\end{array}$ & $\begin{array}{c}\text { Posterior a la decisión tomada } \\
\text { con WRC y CBA. }\end{array}$ \\
\hline Tiempo total por método & $\begin{array}{l}\text { La suma entre el tiempo de } \\
\text { consenso y de método }\end{array}$ & Escala temporal & $\begin{array}{c}\text { Posterior a la decisión tomada } \\
\text { con } \mathrm{S} / \mathrm{M}, \mathrm{WRC} \text { y CBA }\end{array}$ \\
\hline
\end{tabular}


Tabla 2: Satisfacción personal de las diez empresas participantes

\begin{tabular}{|c|c|c|c|c|c|c|c|c|c|c|}
\hline Empresas & A & B & C & D & E & F & G & H & I & J \\
\hline S/M & 9.0 & 9.0 & 9.5 & 8.5 & 10.0 & 10.0 & 10.0 & 10.0 & 10.0 & 9.0 \\
\hline WRC & 9.0 & 10.0 & 8.5 & 10.0 & 10.0 & 9.0 & 10.0 & 8.5 & 10.0 & 9.0 \\
\hline CBA & 9.0 & 9.5 & 10.0 & 10.0 & 10.0 & 10.0 & 9.0 & 9.0 & 10.0 & 10.0 \\
\hline
\end{tabular}

Se puede concluir que el uso del método CBA genera un mayor aumento de satisfacción personal del grupo de trabajo al ser contrastado con los otros dos métodos de selección. Para poder afirmar estadísticamente si dichos aumentos de satisfacción personal son significativos o no, se procede a realizar un análisis estadístico de las respuestas individuales de todos los participantes de la simulación asociado a la satisfacción personal que experimentaron. En primer lugar, se hace uso de la prueba de normalidad de Shapiro-Wilk con un valor de significancia $\alpha$ igual a 0.05 . Para dicha prueba, se contrasta S/M vs WRC, S/M vs CBA y WRC vs CBA mediante el programa computacional SPSS (IBM, 2014). Se determina que los datos analizados no distribuyen normal, por lo cual para realizar el análisis estadístico respectivo se deben usar pruebas no paramétricas. Posteriormente, se hace uso de la prueba U de Mann Whitney para muestras independientes no paramétricas, para determinar si existe o no una diferencia estadísticamente significativa. Para dicha prueba, se asume un valor de significancia $\alpha$ igual a 0.05 . Finalmente, como todos los valores de p-value fueron mayores a 0.05 no se puede afirmar que exista una diferencia estadísticamente significativa entre las satisfacciones personales obtenidas al comparar los métodos expuestos en la simulación. Por lo cual, se concluye que el método CBA genera ligeramente mayores grados de satisfacción personal del grupo de trabajo al ser contrastado con los otros dos métodos de la simulación, es decir, S/M y WRC. Dicha afirmación es respaldada estadísticamente al no existir diferencias significativas entre dichos métodos. Se presume que los grupos de trabajo que participaron en la simulación de toma de decisiones llevan tomando decisiones de manera grupal hace un largo tiempo, ya que los resultados evidencian altos niveles de satisfacción en todos sus participantes, tales niveles de satisfacción personal sólo se logran trabajando en equipo y escuchando la opinión de todos sus miembros. Por lo tanto, el nivel de cultura de equipos del grupo que toma la decisión es importante.
El segundo indicador medido fue la frustración personal, dicho indicador mide qué tan frustrados se sienten con la decisión grupal respecto a su decisión individual. Su escala de medición es de uno a diez, siendo uno el menor grado de frustración y diez el mayor grado de frustración. Los resultados se presentan en la Tabla 3 y representan la mediana de las frustraciones de los trabajadores en cada una de las diez empresas participantes (A-J).

Tabla 3: Frustración personal de las diez empresas participantes

\begin{tabular}{|c|c|c|c|c|c|c|c|c|c|c|}
\hline Empresa & A & B & C & D & E & F & G & H & I & J \\
\hline S/M & 1.0 & 1.0 & 1.0 & 2.5 & 1.0 & 1.0 & 1.0 & 1.0 & 1.0 & 2.0 \\
\hline WRC & 1.5 & 1.5 & 2.5 & 1.0 & 1.0 & 1.0 & 1.0 & 1.0 & 1.0 & 1.0 \\
\hline CBA & 1.5 & 1.0 & 1.0 & 1.0 & 1.0 & 1.0 & 1.0 & 1.5 & 1.0 & 1.0 \\
\hline
\end{tabular}

En primera instancia, se puede concluir que, usando cualquier método expuesto en la simulación, es decir, la forma habitual de decisión de las empresas (S/M), el primer método enseñado WRC o el segundo método enseñado CBA, genera bajos niveles de frustración personal del grupo de trabajo. Se puede concluir que el uso del método WRC o CBA genera la misma disminución de la frustración personal del grupo de trabajo al ser contrastado con el método habitual de decisión de las empresas (S/M).

Para poder afirmar estadísticamente si dichas disminuciones de frustración personal son significativas o no, se procede a realizar un análisis estadístico de las respuestas individuales de todos los participantes de la simulación asociado a la frustración personal que experimentaron. Análogamente, se hace uso de la prueba de normalidad de Shapiro-Wilk con un valor de significancia $\alpha$ igual a 0.05. Para dicha prueba, se contrasta $\mathrm{S} / \mathrm{M}$ vs WRC, S/M vs CBA y WRC vs CBA mediante el programa computacional SPSS. Se determina que los datos analizados no distribuyen normal, por lo cual para realizar el análisis estadístico respectivo se deben usar pruebas no paramétricas. Posteriormente, se hace uso de la prueba no paramétrica U de Mann Whitney para muestras independientes, para determinar si existe o no una diferencia estadísticamente significativa. Para dicha prueba, se asume un valor de significancia $\alpha$ igual a 0.05 . Finalmente, como todos los valores de p-value fueron mayores a 0.05 no se puede afirmar que exista una diferencia estadísticamente significativa entre las 
frustraciones personales obtenidas al comparar los métodos expuestos en la simulación. Por lo cual, se concluye que el uso de cualquier método expuesto en la simulación, es decir, la forma habitual de decisión de las empresas (S/M), el primer método propuesto WRC o el segundo método propuesto CBA, no genera grandes cambios en la frustración personal del grupo de trabajo. Dicha afirmación es respaldada estadísticamente al no existir diferencias significativas entre dichos métodos. Se podría entender que la satisfacción y la frustración son complementarios, ahora bien, para la simulación era importante evaluar ambos para revisar si había consistencia en las respuestas. De igual manera que en el primer indicador, se presume que los grupos de trabajo que participaron en la simulación llevan un buen tiempo decidiendo de manera grupal, ya que exhiben bajos niveles de frustración personal de sus trabajadores, tales niveles de frustración son alcanzados al escuchar la opinión de todos los miembros del grupo de trabajo. El tercer indicador medido fue la dificultad percibida del método, dicho indicador sólo se mide cuando se aplica WRC y CBA. Su escala de medición es de uno a diez, siendo uno el menor grado de dificultad y diez el mayor grado de dificultad. Los resultados se presentan en la Tabla 4 y representan la mediana de las dificultades percibidas de los trabajadores en cada una de las diez empresas participantes (A-J).

Tabla 4: Dificultad percibida del método de las diez empresas participantes.

\begin{tabular}{|c|c|c|c|c|c|c|c|c|c|c|}
\hline Empresa & A & B & C & D & E & F & G & H & I & J \\
\hline WRC & 1.5 & 2.0 & 5.5 & 5.5 & 1.0 & 2.0 & 1.0 & 2.5 & 4.5 & 3.0 \\
\hline CBA & 2.0 & 4.5 & 6.5 & 4.0 & 6.5 & 8.0 & 4.0 & 4.5 & 5.0 & 5.0 \\
\hline
\end{tabular}

Se puede concluir que el método WRC es percibido como más sencillo en su uso y desarrollo al ser contrastado con el método CBA. Para poder afirmar estadísticamente si existe una diferencia significativa en dicho indicador al contrastar los métodos de selección, se procede a realizar un análisis estadístico de las respuestas individuales de todos los participantes de la simulación asociado a la dificultad percibida del método que experimentaron. Análogamente, se hace uso de la prueba de normalidad de Shapiro-Wilk con un valor de significancia $\alpha$ igual a 0.05 . Para dicha prueba, se contrasta WRC vs CBA mediante el programa computacional SPSS. Se determina que los datos analizados no distribuyen normal, por lo cual para realizar el análisis estadístico respectivo se debe usar una prueba no paramétrica.

Posteriormente, se hace uso de la prueba U de Mann Whitney para muestras independientes, para determinar si existe o no una diferencia estadísticamente significativa. Para dicha prueba, se asume un valor de significancia $\alpha$ igual a 0.05 y mediante el programa computacional SPSS se obtiene un valor p-value de 0.005 , por lo cual se interpreta que sí existen diferencias significativas entre dichos datos.

Finalmente, se concluye que el método WRC es percibido como más sencillo en su uso y desarrollo al ser contrastado con el método CBA y dicha afirmación es respaldada estadísticamente al comprobar la existencia de diferencias significativas entre dichos métodos.

Se presume que el método WRC es percibido como un método más fácil, ya que para los participantes de la simulación fue un método más cercano a la realidad. De hecho, más de la mitad de los participantes, inconscientemente, desarrollaban una estructura de pasos parecida a la del método WRC, es decir, ponderaban las características de una forma subjetiva y luego calificaban dicha característica por cada opción a elegir. En cambio, el método CBA poseía una estructura de pasos totalmente nueva, por lo cual a los participantes les costaba más asimilar dichos pasos y ejecutarlos posteriormente. Se intuye que la dificultad percibida de ambos métodos debería igualarse o al menos acercarse, una vez dichos métodos se coloquen en práctica por los grupos de trabajo.

El cuarto indicador medido fue la probabilidad de implementación del método, dicho indicador se mide una vez que los participantes hayan aplicado los métodos propuestos, en este caso, WRC y CBA. Su escala de medición es de uno a diez, siendo uno el menor grado de probabilidad de implementación y diez el mayor grado de probabilidad de implementación, para efectos de análisis se usaron dichas mediciones en escala de 0 a 1 . Los resultados se presentan en la Tabla 5 y representan la mediana de las probabilidades de implementación del método de los trabajadores de las diez empresas participantes (A-J). 
Tabla 5: Probabilidad de implementación del método de las diez empresas participantes

\begin{tabular}{|c|c|c|c|c|c|c|c|c|c|c|}
\hline Empresa & A & B & C & D & E & F & G & H & I & J \\
\hline WRC & 0.70 & 0.65 & 0.70 & 0.65 & 0.85 & 0.70 & 0.85 & 0.90 & 0.80 & 0.70 \\
\hline CBA & 0.75 & 0.55 & 0.55 & 0.85 & 0.15 & 0.30 & 0.55 & 0.70 & 0.85 & 0.55 \\
\hline
\end{tabular}

Se puede concluir que el método WRC obtiene mayor probabilidad de implementación en las empresas al ser contrastado con el método CBA. Para poder afirmar estadísticamente si existe una diferencia significativa en dicho indicador al contrastar los métodos de selección, se procede a realizar un análisis estadístico de las respuestas individuales de todos los participantes de la simulación asociado a la probabilidad de implementación del método que experimentaron. Análogamente, se hace uso de la prueba de normalidad de Shapiro-Wilk con un valor de significancia $\alpha$ igual a 0.05 . Para dicha prueba, se contrasta WRC vs CBA mediante el programa computacional SPSS. Se determina que los datos analizados nodistribuyen normal, por lo cual para realizar el análisis estadístico respectivo se debe usar una prueba no paramétrica. Posteriormente, se hace uso de la prueba no paramétrica U de Mann Whitney para muestras independientes, para determinar si existe o no una diferencia estadísticamente significativa. Para dicha prueba, se asume un valor de significancia $\alpha$ igual a 0.05 y mediante el programa computacional SPSS se obtiene un valor $\mathrm{p}$-value de 0.063 , por lo cual se interpreta que no existen diferencias significativas entre dichos datos. Finalmente, se concluye que el método WRC es valorado con una mayor probabilidad de implementación en las empresas al ser contrastado con el método CBA. Se presume que el método WRC es valorado con una mayor probabilidad de implementación en las empresas, ya que es percibido como un método más fácil y segundo, porque posee una estructura de pasos sencilla y familiar para los participantes del experimento de simulación.

El quinto indicador medido fue el tiempo empleado en usar el método, dicho indicador se mide en una escala temporal, donde el inicio se define como el momento en que empiezan a usar el método y su final como el momento en que todos los participantes toman su decisión individual. Los resultados se presentan en la Tabla 8 y representan los tiempos empleados para usar el método en cada una de las diez empresas participantes (A-J).
Tabla 8: Tiempo del método de las diez empresas participantes.

\begin{tabular}{|c|c|c|c|c|c|c|c|c|c|c|}
\hline Empresa & $\mathrm{A}$ & $\mathrm{B}$ & $\mathrm{C}$ & $\mathrm{D}$ & $\mathrm{E}$ & $\mathrm{F}$ & $\mathrm{G}$ & $\mathrm{H}$ & $\mathrm{I}$ & $\mathrm{J}$ \\
\hline S/M & $0: 03: 50$ & $0: 02: 31$ & $0: 04: 38$ & $0: 03: 50$ & $0: 02: 48$ & $0: 05: 10$ & $0: 08: 36$ & $0: 03: 10$ & $0: 02: 32$ & $0: 03: 58$ \\
\hline WRC & $0: 13: 00$ & $0: 20: 26$ & $0: 11: 34$ & $0: 16: 45$ & $0: 11: 37$ & $0: 14: 49$ & $0: 17: 22$ & $0: 15: 05$ & $0: 08: 24$ & $0: 14: 01$ \\
\hline CBA & $0: 17: 39$ & $0: 25: 47$ & $0: 20: 10$ & $0: 22: 25$ & $0: 16: 23$ & $0: 22: 43$ & $0: 17: 24$ & $0: 16: 35$ & $0: 13: 33$ & $0: 18: 59$ \\
\hline
\end{tabular}

Se puede concluir que usando la forma habitual de decisión (S/M) los tiempos de empleo son menores que con los métodos WRC y CBA. Por otra parte, los tiempos de empleo del método WRC son menores que con el método CBA. Para poder afirmar estadísticamente si existe una diferencia significativa en dicho indicador al contrastar los métodos de selección, se procede a realizar un análisis estadístico de las respuestas individuales de todos los participantes de la simulación asociado al tiempo empleado en usar los métodos que experimentaron. Análogamente, se hace uso de la prueba de normalidad de Shapiro-Wilk con un valor de significancia $\alpha$ igual a 0.05 . Para dicha prueba, se contrasta $\mathrm{S} / \mathrm{M}$ vs WRC, S/M vs CBA y WRC vs CBA mediante el programa computacional SPSS. Se determina que en las dos primeras comparaciones los datos analizados no vienen de una distribución normal, por lo cual para realizar el análisis estadístico respectivo se deben usar pruebas no paramétricas. En cambio, al comparar WRC vs CBA los datos analizados vienen de una distribución normal, por lo cual para realizar el análisis estadístico respectivo se debe usar una prueba paramétrica.

Se utiliza la prueba paramétrica t-Student y la prueba no paramétrica U de Mann Whitney, dependiendo el caso, y se asumen muestras independientes, esto con el fin de determinar si existe o no una diferencia estadísticamente significativa. Cabe destacar, que inicialmente se verifica la igualdad de varianzas de las muestras para el adecuado uso de la prueba t-Student. Para dichas pruebas, se asume un valor de significancia $\alpha$ igual a 0.05 y mediante el programa computacional SPSS, se obtienen los resultados mostrados en la Tabla 9.

Tabla 9: Resultados del test t-Student y test U de Mann Whitney asociados al tiempo empleado en el uso del método

\begin{tabular}{|c|c|c|c|}
\hline Métodos & p-value & H0 & Conclusión \\
\hline S/M vs WRC & 0.000 & Rechazada & $\begin{array}{c}\text { Sí hay diferencias } \\
\text { significativas }\end{array}$ \\
\hline S/M vs CBA & 0.000 & Rechazada & $\begin{array}{c}\text { Sí hay diferencias } \\
\text { significativas }\end{array}$ \\
\hline WRC vs CBA & 0.070 & $\begin{array}{c}\text { No se puede } \\
\text { rechazar }\end{array}$ & $\begin{array}{c}\text { No hay diferencias } \\
\text { significativas }\end{array}$ \\
\hline
\end{tabular}


Finalmente, se puede afirmar que exista una diferencia estadísticamente significativa de los tiempos empleados en usar el método al contrastar S/M con WRC y al contrastar $\mathrm{S} / \mathrm{M}$ con $\mathrm{CBA}$. En cambio, no existe una diferencia estadísticamente significativa de dicho indicador al contrastar WRC con CBA. Por lo cual, se concluye que el método habitual de selección de las empresas $(\mathrm{S} / \mathrm{M})$ es más rápido en su uso al ser contrastado con los dos métodos propuestos, es decir, WRC y CBA. Dicha afirmación es respaldada estadísticamente al comprobar la existencia de diferencias significativas entre dichos métodos. Se presume que la rapidez exhibida en el método habitual de selección de las empresas $(\mathrm{S} / \mathrm{M})$ se debe a que dicho método no posee ninguna estructura de pasos, por lo cual al agregar una estructura definida de pasos como en los dos métodos propuestos, es decir, WRC y CBA, se aumentan inevitablemente los tiempos para tomar decisiones.

El sexto indicador medido fue el tiempo en alcanzar un consenso, dicho indicador se mide en una escala temporal, donde el inicio se define como el momento en que empiezan a discutir y argumentar sus decisiones para convencer a los demás participantes y su final se define como el momento en que se alcanza dicho consenso. Los resultados se presentan en la Tabla 10 y representan los tiempos en alcanzar el consenso en cada una de las diez empresas participantes (A-J).

Tabla 10: Tiempo en alcanzar el consenso de las diez empresas participantes

\begin{tabular}{|c|c|c|c|c|c|c|c|c|c|c|}
\hline Empresa & A & B & C & D & E & F & G & H & I & J \\
\hline S/M & $0: 02: 58$ & $0: 00: 49$ & $0: 01: 45$ & $0: 01: 34$ & $0: 00: 58$ & $0: 10: 35$ & $0: 00: 57$ & $0: 01: 00$ & $0: 01: 47$ & $0: 02: 00$ \\
\hline WRC & $0: 04: 17$ & $0: 01: 56$ & $0: 11: 27$ & $0: 01: 01$ & $0: 02: 47$ & $0: 12: 42$ & $0: 01: 47$ & $0: 00: 53$ & $0: 04: 28$ & $0: 04: 10$ \\
\hline CBA & $0: 01: 56$ & $0: 00: 42$ & $0: 01: 30$ & $0: 00: 48$ & $0: 00: 30$ & $0: 00: 52$ & $0: 06: 58$ & $0: 00: 25$ & $0: 03: 23$ & $0: 01: 37$ \\
\hline
\end{tabular}

Se puede concluir que usando el método CBA disminuyen los tiempos para alcanzar los consensos en los grupos de trabajo al ser comparados con los otros dos métodos expuestos en la simulación, es decir, S/M y WRC. Para poder afirmar estadísticamente si existe una diferencia significativa en dicho indicador al contrastar los métodos de selección, se procede a realizar un análisis estadístico de las respuestas individuales de todos los participantes de la simulación asociado al tiempo en alcanzar consensos que experimentaron los grupos de trabajo. Análogamente, se hace uso de la prueba de normalidad de Shapiro-Wilk, esto con el fin de precisar si se deben usar pruebas paramétricas o no paramétricas. Para dicha prueba, se contrasta S/M vs WRC, S/M vs CBA y WRC vs CBA mediante el programa computacional SPSS. Se determina que los datos analizados no vienen de una distribución normal, por lo cual para realizar el análisis estadístico respectivo se deben usar pruebas no paramétricas. Posteriormente, se hace uso de la prueba $U$ de Mann Whitney para muestras independientes, para determinar si existe o no una diferencia estadísticamente significativa. Para dicha prueba, se asume un valor de significancia $\alpha$ igual a 0.05 y mediante el programa computacional SPSS, se obtienen los resultados mostrados en la Tabla 11.

Tabla 11: Resultados del test U de Mann Whitney asociados al tiempo para alcanzar consensos

\begin{tabular}{|l|l|l|l|}
\hline \multicolumn{1}{|c|}{ Métodos } & p-value & \multicolumn{1}{c|}{ H0 } & \multicolumn{1}{c|}{ Conclusión } \\
\hline S/M vs WRC & 0.076 & $\begin{array}{l}\text { No se puede } \\
\text { rechazar }\end{array}$ & $\begin{array}{l}\text { No hay diferencias } \\
\text { significativas }\end{array}$ \\
\hline S/M vs CBA & 0.257 & $\begin{array}{l}\text { No se puede } \\
\text { rechazar }\end{array}$ & $\begin{array}{l}\text { No hay diferencias } \\
\text { significativas }\end{array}$ \\
\hline $\begin{array}{l}\text { WRC vs } \\
\text { CBA }\end{array}$ & 0.026 & Rechazada & $\begin{array}{l}\text { Sí hay diferencias } \\
\text { significativas }\end{array}$ \\
\hline
\end{tabular}

Finalmente, se puede afirmar que exista una diferencia estadísticamente significativa de los tiempos para alcanzar consensos al contrastar WRC con CBA. En cambio, no existe una diferencia estadísticamente significativa de dicho indicador al contrastar $\mathrm{S} / \mathrm{M}$ con WRC y $\mathrm{S} / \mathrm{M}$ con CBA. Por lo cual, se concluye que el método CBA es más rápido para alcanzar consensos en los grupos de trabajo al ser contrastado con el método WRC y es ligeramente más rápido al ser contrastado con el método habitual de las empresas ( $\mathrm{S} / \mathrm{M})$. La primera afirmación es respaldada estadísticamente al comprobar la existencia de diferencias significativas entre los métodos CBA y WRC. Y la segunda afirmación es respaldada estadísticamente al no existir diferencias significativas entre los métodos CBA y $\mathrm{S} / \mathrm{M}$. Se presume que la mayor rapidez exhibida para alcanzar consensos del método CBA, se debe a que el gráfico final de dicho método da la posibilidad de tomar una postura individual al principio y de poder entablar una discusión con dicha herramienta visual para lograr mejores decisiones grupales. En cambio, en los otros dos métodos no se posee dicha herramienta visual, por lo cual es más difícil convencer a la otra persona para llegar a un consenso. 
El séptimo indicador medido fue el tiempo total por método, dicho indicador se mide en una escala temporal, donde el inicio se define como el momento en que empiezan a usar el método y su final se define como el momento que los participantes llegan a un consenso. Como dicho indicador es la suma del tiempo de método y el tiempo de consenso, el análisis estadístico realizado nos indica que sus resultados se comportan de la misma manera que el indicador de tiempo de método, esto ocurre ya que los tiempos de método predominan sobre los tiempos de consenso al ser mayor de magnitud. Finalmente, se concluye que el método habitual de selección de las empresas ( $\mathrm{S} / \mathrm{M})$ es más rápido en su uso al ser contrastado con los dos métodos propuestos, es decir, WRC y CBA. Dicha afirmación es respaldada estadísticamente al comprobar la existencia de diferencias significativas entre dichos métodos. Para una mejor visualización de los resultados anteriormente expuestos, se presenta un resumen de resultados en la Tabla 12.

Tabla 12: Resumen de resultados

\begin{tabular}{|l|c|}
\hline Indicador analizado & $\begin{array}{c}\text { Método con mejor } \\
\text { desempeño }\end{array}$ \\
\hline Satisfacción personal & CBA \\
\hline Frustración personal & - \\
\hline Dificultad percibida del método & WRC \\
\hline Probabilidad de implementación del método & WRC \\
\hline Tiempo empleado en usar el método & WRC* \\
\hline Tiempo en alcanzar un consenso & CBA \\
\hline Tiempo total por método & WRC* \\
\hline
\end{tabular}

* En estos casos, cabe precisar que el método con mejor desempeño fue el método S/M. Sin embargo, para contrastar los dos métodos multicriterios propuestos (WRC y CBA) se optó por definir el método con mejor desempeño entre dichos métodos.

Se puede observar claramente, que el método WRC se desempeñó de mejor manera al ser contrastado con el método CBA en los análisis anteriores. Por lo cual, dicho método será recomendado para usar en la metodología de toma de decisiones final.

\section{Metodología de toma de decisiones propuesta}

A continuación, se presenta la metodología de toma de decisiones para seleccionar subcontratistas en la industria de la construcción.

\section{Objetivo}

Proporcionar un instrumento técnico-administrativo, traducido en un manual tentativo, para la óptima selección de subcontratistas en la industria de la construcción.

\section{Alcances}

Subcontratos asociados al rubro de la construcción, por ejemplo: partidas de acero, moldajes, hormigón, tabiquería, mecánica de suelos, etc.

\section{Recomendaciones antes del proceso de selección}

Antes del proceso de selección, la empresa debe definir los posibles subcontratos que cumplirán los requerimientos del proyecto a construir. En dicho escenario, la empresa debe recopilar la mayor cantidad de información de los posibles subcontratos, de esta manera, se podrán analizar de mejor manera y abarcando un mayor número de variables que intervienen en la decisión.

\section{Método sugerido - WRC}

A continuación, se presentan los pasos y recomendaciones para el uso del método multicriterio de toma de decisiones WRC:

1. Determinar las alternativas de selección. Se deben definir todas las posibles alternativas de subcontratos que cumplan con los requerimientos mínimos del proyecto a realizar.

2. Determinar los factores que se evaluarán en el proceso de selección. Se deben definir todos los factores importantes para la empresa a la hora de tomar la decisión (costos, calidad, cumplimiento de plazos, solvencia económica, tasa de accidentes, entre otros).

3. Decidir los pesos de cada factor. Se deben definir los pesos para cada factor según el proyecto en particular, es decir, los pesos pueden variar entre proyectos. Se recomienda llegar a consensos en los grupos de trabajo respecto a los pesos de cada factor, de esta manera, la decisión final debería satisfacer a todos los participantes de la selección (obs: la suma de los pesos debe ser 100\%).

4. Resumir las características de cada subcontratista asociado a cada factor. Se debe resumir las características de cada subcontratista asociado a 
cada factor, de esta manera, se podrán evidenciar las diferencias entre las alternativas de selección.

5. Asignar un puntaje a las características de cada subcontratista: se deben asignar puntajes numéricos de uno a diez, siendo uno el menor puntaje y diez el mayor puntaje a cada característica resumida en el punto anterior. Se recomienda llegar a consensos en los grupos de trabajo respecto a los puntajes asignados, de esta manera, la decisión final debería satisfacer a todos los participantes de la selección.

6. Calcular el puntaje total de cada subcontratista. Se debe calcular el puntaje total de cada subcontratista, de la siguiente manera:

Puntaje total $=\sum$ (Peso del factor $)_{\mathrm{i}}$ Puntaje $_{\mathrm{i}} \mathrm{i}$ : cada factor en la decisión (1)

Se recomienda automatizar dicho cálculo de los puntajes totales, de esta manera, se podrán visualizar posibles cambios en la decisión, según se ajusten los pesos iniciales en una determinada situación.

7. Seleccionar el mejor subcontratista. El mayor puntaje total de todas las alternativas será el subcontrato óptimo para la empresa, según todas las decisiones tomadas anteriormente.

Finalmente, la Tabla 13 presenta una tabla tentativa para el uso del método sugerido WRC.

\section{Conclusión}

Una vez realizada la revisión del estado de la subcontratación en la industria de la construcción, tanto a nivel país como a nivel mundial, se puede concluir que: la estrategia de negocios de subcontratar es ampliamente usada en el rubro, ya que la construcción es una rama compleja y son pocas las empresas que pueden realizar todos los trabajos, o partidas requeridas, con una buena calidad, cumpliendo los plazos estipulados y, al mismo tiempo, generando utilidades. En la actualidad, la selección de subcontratación se lleva a cabo de una manera precaria, es decir, no hay metodologías claras para seleccionar los subcontratos en cada proyecto. Según el diagnóstico de las prácticas actuales de subcontratación, el $46 \%$ de las empresas manifestó que la característica más valorada al subcontratar es la experiencia previa con sus subcontratistas, es decir, cada empresa posee una base de datos interna de subcontratos que han trabajado con ellos. Dicha base de datos sería la metodología para seleccionar subcontratistas que tienen las empresas, ya que en un futuro proyecto sólo deben contactar al subcontrato que se ha desempeñado adecuadamente en el pasado. Dicha metodología representa un problema para los subcontratistas emergentes, ya que, al ser nuevos en el rubro, deben esperar a que una empresa les brinde la oportunidad de trabajar con ellos en primera instancia. El mismo diagnóstico, indicó que el $66 \%$ de las empresas participantes toman la decisión de subcontratar de manera grupal, en otras palabras, escuchando la opinión de su grupo de trabajo. Para seguir la misma línea, se propusieron dos métodos de toma de decisiones, WRC y CBA, en los cuales los participantes debían usar dichos métodos de forma individual y luego debían llegar a consensos grupales.

El experimento de simulación de toma de decisiones dio la posibilidad de determinar cuál de los dos métodos propuestos se desempeñó de mejor manera en dicha experiencia, esto con el fin de incluir dicho método en la metodología final de la presente investigación. Para determinar el mejor método, se midieron y analizaron siete indicadores. Los resultados fueron concluyentes, ya que

Tabla 13: Tabla propuesta para el uso del método sugerido - WRC

\begin{tabular}{|c|c|c|c|c|c|c|c|}
\hline \multicolumn{8}{|c|}{ Decisión según WRC } \\
\hline \multirow{2}{*}{ Factor } & \multirow{2}{*}{$\begin{array}{l}\text { Peso del } \\
\text { factor }\end{array}$} & Subcontratista 1 & \multirow{2}{*}{ Puntaje 1} & Subcontratista 2 & \multirow{2}{*}{$\begin{array}{c}\text { Puntaje } \\
2\end{array}$} & Subcontratista 3 & \multirow{2}{*}{$\begin{array}{c}\text { Puntaje } \\
3\end{array}$} \\
\hline & & Calificación & & Calificación & & Calificación & \\
\hline & & & & & & & \\
\hline & & & & & & & \\
\hline & & & & & & & \\
\hline & & & & & & & \\
\hline & & & & & & & \\
\hline Puntaje total & $100 \%$ & - & & - & & - & \\
\hline
\end{tabular}


el método WRC se desempeñó mejor que el método CBA en cuatro de los siete indicadores individuales (dificultad percibida del método, probabilidad de implementación del método, tiempo del método y tiempo total del método).

Se concluye que el método WRC es el método adecuado para seleccionar subcontratistas en la industria de la construcción, ya que es percibido como un método simple, fácil de implementar en las empresas y además posee bajos tiempos de ejecución. Dicho método se incluye en la metodología de toma de decisiones propuesta. Los experimentos de simulación se hicieron bajo el supuesto implícito que existían varias alternativas de subcontrato, lo que no pasa necesariamente en lugares aislados en donde no existen varias opciones para elegir.

Para futuras investigaciones se recomienda evaluar los métodos de selección de subcontratos en equipos con personas con diferentes grados de conocimiento entre ellos, de esta forma, se podrá evidenciar lo importante que es armar equipos en que exista confianza y cohesión.

\section{Referencias}

Alvarado, A. y Spolmann, S. (2009). Análisis de competencia del sector de la construcción chileno y sus procesos de licitaciones públicas de contrato de obras: estructuras, agentes y prácticas. Fiscalía Nacional Económica, Gobierno de Chile, Santiago, http://www.fne.gob.cl/wpcontent/uploads/2011/03/estu_0001_2009.pdf, visitado 15/10/2017

Arroyo, P., Fuenzalida, C., Albert, A. and Hallowell, M.R. (2016). Collaborating in decision making of sustainable building design: an experimental study comparing CBA and WRC methods. Energy and Buildings 128, 132-142

Arroyo, P., Tommelein, I.D. and Ballard, G. (2014). Comparing weighting rating and calculating vs. choosing by advantages to make design choices. $22^{\text {nd }}$ Annual Conference of the International Group for Lean Construction IGLC, Oslo, Norway, 401-412

Assaf, S.A. and Al-Hejji, S. (2006). Causes of delay in large construction projects. International Journal of Project Management 24(4), 349-357

Assaf, S.A., Al-Khalil, M. and Al-Hazmi, M. (1995). Causes of delays in large building construction projects. Journal of Management in Engineering 11(2), 45-50

Grant, E.J. and Jones, J.R. (2008). A decision-making framework for vegetated roofing system selection. Journal of Green Building 3(4), $138-153$
Harris, R. (2012). Introduction to decision making. http://www. virtualsalt.com/crebook5.htm, visitado 15/11/2017

IBM (2014). Statistical Package for the Social Sciences SPSS. IBM SPSS Statistics. New York, USA

Kleindorfer, P.R., Kunreuther, H.G. and Schoemaker, P.J.H. (1993). Decision sciences: an integrative perspective. Cambridge University Press, UK

Mezher, T.M. and Tawil, W. (1998). Causes of delays in the construction industry in Lebanon. Engineering, Construction and Architectural Management 5(3), 252-260

Nguyen, H.V., Lostuvali, B. and Tommelein, I.D. (2009). Decision analysis using virtual first-run study of a viscous damping wall system. 17 th Annual Conference of the International Group for Lean Construction IGLC, Taipei, Taiwan, 371-382

Odeh, A.M. and Battaineh, H.T. (2002). Causes of construction delay: traditional contracts. International Journal of Project Management 20(1), 67-73

OIT (2007). El trabajo en régimen de subcontratación en América Latina. Oficina Internacional del Trabajo OIT, Ginebra, Suiza

Ogunlana, S.O., Promkuntong, K., Jearkjirm, V. (1996). Construction delays in fast-growing economy: comparing Thailand with other economies. International Journal of Project Management 14(1), 37-45

Sabapathy, A. and Maithel, S. (2013). A multi-criteria decision analysis based assessment of walling materials in India. Building and Environment 64, 107-117

Sambasivan, M. and Soon, Y.W. (2007). Causes and effects of delays in Malaysian construction industry. International Journal of Project Management 25(5), 517-526

Schöttle, A., Arroyo, P. and Bade, M. (2015). Comparing three methods in the tendering procedure to select the project team. $23^{\text {rd }}$ Annual Conference of the International Group for Lean Construction IGLC, Perth, Australia, 267-276

Tatum, C. (1984). Organizing large projects: how managers decide. Journal of Construction Engineering and Management 110(3), 346-358

Tzeng, G.H. and Huang, J.J. (2011). Multiple attribute decision making. Methods and applications. CRC Press, Taylor \& Francis Group, USA Uquillas, C.A. (2007). El outsourcing en el Ecuador. Observatorio de la Economía Latinoamericana 87. http://www.eumed.net/cursecon/ecolat/ index.htm, visitado 14/11/2017 